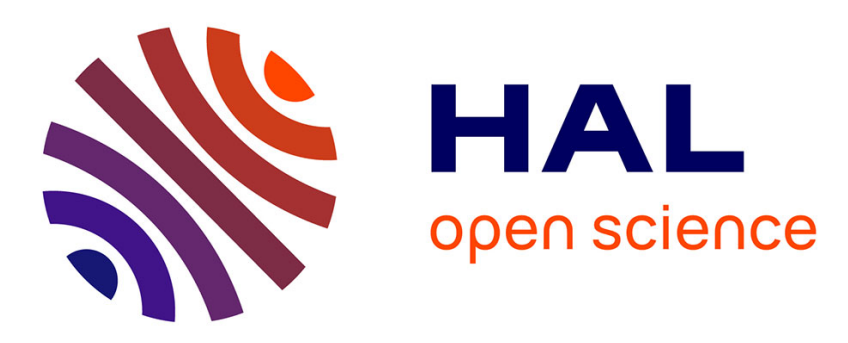

\title{
Mesh adaptation for large-eddy simulations in complex geometries
}

\author{
Pierre Benard, Guillaume Balarac, Vincent Moureau, Cécile Dobrzynski, \\ Ghislain Lartigue, Yves d'Angelo
}

\section{- To cite this version:}

Pierre Benard, Guillaume Balarac, Vincent Moureau, Cécile Dobrzynski, Ghislain Lartigue, et al.. Mesh adaptation for large-eddy simulations in complex geometries. International Journal for Numerical Methods in Fluids, 2016, 81 (12), pp.719-740. 10.1002/fld.4204 . hal-01339519

\section{HAL Id: hal-01339519 \\ https://hal.science/hal-01339519}

Submitted on 26 Apr 2019

HAL is a multi-disciplinary open access archive for the deposit and dissemination of scientific research documents, whether they are published or not. The documents may come from teaching and research institutions in France or abroad, or from public or private research centers.
L'archive ouverte pluridisciplinaire HAL, est destinée au dépôt et à la diffusion de documents scientifiques de niveau recherche, publiés ou non, émanant des établissements d'enseignement et de recherche français ou étrangers, des laboratoires publics ou privés. 


\title{
Mesh adaptation for large-eddy simulations in complex geometries
}

\author{
P. Benard ${ }^{1 *}$, G. Balarac ${ }^{2}$, V. Moureau ${ }^{1}$, C. Dobrzynski ${ }^{3}$, G. Lartigue $^{1}$, Y. D’Angelo ${ }^{1}$ \\ ${ }^{1}$ CORIA-UMR6614, Normandie Université, CNRS, INSA and Université de Rouen, Av. de l'Université, 76800 Saint \\ Etienne du Rouvray, France \\ ${ }^{2}$ Univ. Grenoble Alpes/CNRS, LEGI UMR 5519, F-38000 Grenoble, France
}

${ }^{3}$ Bordeaux INP, IMB, UMR 5251, F-33400, Talence, France and Inria Bordeaux Sud-Ouest, Team CARDAMOM, 33405

Talence, France

SUMMARY

Large-eddy simulation (LES) consists in explicitly simulating the large scales of the fluid motion and in modeling the influence of the smallest scales. Thanks to the steady growth of computational resources, LES can now be used to simulate realistic systems with complex geometries. However, when LES is used in such complex geometries, an adequate mesh has to be determined to perform valid LES. In this work, a strategy is proposed to assess the quality of a given mesh and to adapt it locally. Two different criteria are used as mesh adaptation criteria. The first criterion is defined to ensure a correct discretization of the mean field, whereas the second criterion is defined to ensure enough explicit resolution of turbulent scales motions. The use of both criteria are shown in canonical flow cases. As a second part of this work, a numerical strategy for mesh adaptation in HPC context is proposed by coupling the flow solver, YALES2, and the remeshing library, MMG3D, for massively parallel computations. This coupling enables an efficient and parallel remeshing of grids alleviating any memory or performance issues encountered in sequential tools. This strategy is finally applied to the simulation of the iso-thermal flow in a complex meso-combustor to demonstrate the applicability of the adaptation methodology to complex turbulent flows. Copyright (C) 2010 John Wiley \& Sons, Ltd.

Received ...

KEY WORDS: LES quality criteria; Parallel mesh adaptation; Adaptation procedure; Meso-scale application 


\section{INTRODUCTION}

Due to the large range of scale motions, a direct numerical simulation (DNS) of turbulent flows is often not possible in realistic applications. To allow a numerical prediction of such flows, the large-eddy simulation (LES) technique appears as an efficient approach. Indeed, LES proposes to explicitly solve the largest scales of the flow and to model the smallest and most computational expensive scales. This scales separation is formally performed by a filtering operation, with a filtering operator characterized by a filter width, $\Delta$. The filtering operation applied to the non-linear term of the Navier-Stokes equations leads to sub-filter terms which should be modeled to perform LES. This filtering operation can be applied explicitly by filtering the non-linear terms at each time step allowing independence between the filter width and the mesh size [1]. But, in majority of CFD codes, this filtering operation is performed implicitly by the mesh size and it is assumed that the filtering width and the mesh size are equal. The sub-filter terms to model are then called sub-grid scale (SGS) term and it is now dependent of the mesh size. The consequence is that the influence of the SGS model decreases with the mesh size and that grid independency can then not be achieved in LES: in the case where the mesh size allows to solve for all the range of turbulent scales, the SGS model becomes negligible and the LES tends towards a DNS. The LES approach can now be used to simulate realistic system in complex geometries, in high performance computing (HPC) context. However, when LES is used in complex geometries, an adequate mesh has to be determined to limit the computational cost while guaranteeing the validity of the sub-grid scale closures.

Various works have already dealt with the assessment of LES quality with the mesh resolution [2, 3]. Various ways have been proposed. Based on previous RANS simulations, the mesh size can be compared to a RANS evaluation of the integral length scale [4] or of the Kolmogorov scale [5]. Theses approaches can be interesting in term of computational cost, but they stay very dependent of the accuracy of the RANS solution. Other strategy is based on systematic grid variation $[6,7]$. In this context, Celik et al. [8,9] propose the Index of Quality (LES_IQ) to examine the LES 
quality. Theses approaches allow a rigorous consideration of both numerical and modeling errors if systematic model variation is also considered. However, these approaches are difficult to use in a systematic way due to the computational cost. Moreover, these approaches needs to consider different but geometrically similar grids, whereas in complex flow configuration strongly nonuniform grid should be considered. Finally, a last strategy based on only one LES calculation appears as a good compromise. Various "single-grid" estimators have been proposed. The simplest one is based on the SGS (or effective) and molecular viscosity ratio [8]. But the recommended value is dependent of the flow [2]. Other quantities can be considered as the ratio between the modeled dissipation and the total (modeled and molecular dissipation) [10] or two-point correlations [11]. Finally, Pope [12] proposes to consider the ratio between the resolved and total turbulent kinetic energy, showing that well-resolved LES should resolve at least $80 \%$ of the total turbulent kinetic energy.

These previous works provide interesting criteria to assess the mesh quality in LES computation, but only few works [13] propose an explicit procedure to determine optimal mesh size for LES and then an automatic mesh adaptation strategy. Moreover, in these previous works, the proposed indicators are mainly to evaluate the part of unresolved (SGS) fields, but other aspects should be taken into account in realistic applications. Then, the objective of this work is to propose a strategy to assess the quality and to adapt a given mesh leading to user-independent meshing. Note that mesh adaptation is an interesting tool for LES modeling but also for time-dependant applications [14, 15] or shock-wave problems $[16,17]$.

The paper is organized as follows: the next section presents the mesh adaptation criteria and their use in canonical flow cases. Section 3 details a numerical strategy for mesh adaptation in HPC context by coupling the flow solver, YALES2, and the mesh adaptation library MMG3D for massively parallel computations. In section 4, this strategy is finally applied to the simulation of the iso-thermal flow in a meso-combustor. The gain in term of computational cost is considered in comparison with a classic homogeneous refinement approach. Conclusions are drawn and work perspectives are proposed in the final section 5 . 


\section{MESH ADAPTATION CRITERIA}

\subsection{Definition of criteria}

As stated in the introduction, grid-independent LES can not be expected for CFD code based on implicit filtering approach. A procedure has then to be defined to assess the quality and to adapt a given mesh, when LES is used to simulate flow in complex geometries. The LES resolved field can be divided in the mean field and the resolved turbulent field, by using a Reynolds decomposition. The mean field is the same field resolved with the RANS approach. The mesh adaptation procedure should ensure a correct resolution of the whole resolved field. From the Reynolds decomposition, it means that the procedure should guarantee (i) an accurate resolution of the mean field and (ii) to explicitly resolve enough turbulent scales motions. Two distinct criteria are then used.

A first criterion has to be defined to ensure a correct resolution of the mean part of the LES resolved field. This criterion is defined to minimize the discretization error of the mean flow gradients. A well known example of this constraint is for the wall-bounded turbulent flows, where a fine mesh has to be used in the direction normal to the wall to accurately predict the strong mean velocity gradient in this direction. Note that this criterion is not specific to LES approach but also exists in RANS approach. In this work, the criterion is defined from an error estimation already proposed for mesh adaptation methodology $[18,19,16]$. From this error estimation, it can be shown that the error between the interpolation, $g^{*}$, of a quantity, $g$, on a discrete space with a size mesh $\Delta$ is limited by a quantity, $Q c_{1}$, defined as,

$$
Q c_{1}=\Delta^{2} \max _{i=1,2,3}\left\{\left|\frac{\partial^{2} g^{*}}{\partial x_{i}^{2}}\right|\right\} .
$$

In this equation we consider the maximal value of the second derivative in each of the three spatial directions. Note also that in the case where this procedure is applied to a vector field $\mathbf{u}$ instead of a scalar field, the Eq. 1 is modified in the following way:

$$
Q c_{1}=\Delta^{2} \max _{i, j=1,2,3}\left\{\left|\frac{\partial^{2} \mathbf{u}_{j}^{*}}{\partial x_{i}^{2}}\right|\right\} .
$$


In this case, the maximum ranges not only on the derivation operator directions but also on the vector field components. The first criterion will be then defined as the minimization of $Q c_{1}$ applied to the mean velocity field in all the computational domain. Specific interpretation of this criterion can be given in LES context. Considering the time-average of an LES resolved velocity component, $\langle\bar{u}\rangle$, this criterion means that the filtering of the average of the resolved field, $\overline{\langle\bar{u}\rangle}=\langle\bar{u}\rangle$ should be close to the average of the resolved field, $\langle\bar{u}\rangle$, because the mean field will not have sub-grid scales contribution. Indeed, in the case of an isotropic filter, a Taylor series expansion of the filtering operator [20] leads to

$$
\langle\bar{u}\rangle=\langle\bar{u}\rangle+\frac{\Delta^{2}}{24} \frac{\partial^{2}\langle\bar{u}\rangle}{\partial x_{i} \partial x_{i}}+\mathcal{O}\left(\Delta^{4}\right),
$$

which leads to the minimization of $\Delta^{2} \partial^{2} \cdot / \partial x_{i}^{2}$, as stated by the first criterion.

A second criterion has to be defined to ensure that a sufficient part of turbulent scales is explicitly resolved to guarantee that LES approach is used in its validity domain [8]. To be valid, the smallest resolved scales have to be far enough to the largest anisotropic scales. They have to be located in the inertial range, where an universal behavior of the scales transfer is expected, allowing to provide accurate modeling. Considering a fully developed turbulence with a classic Kolmogorov spectrum, it can be shown that enough scales are resolved if more than $80 \%$ of the total turbulent kinetic energy is explicitly resolved [12]. The second criterion can then be defined with a quantity $Q c_{2}$ as [21],

$$
Q c_{2}=\frac{E_{\text {sgs }}}{E_{\text {sgs }}+E_{R}} \leq 0.2
$$

with $E_{\text {sgs }}$ the SGS turbulent kinetic energy and $E_{R}=1 / 2\left\langle\bar{u}_{i}^{\prime} \bar{u}_{i}^{\prime}\right\rangle$, the resolved turbulent kinetic energy. $E_{s g s}$ is not explicitly known in LES, but it can be evaluated from the eddy viscosity, $\nu_{s g s}$, given by the SGS model as [22],

$$
E_{\text {sgs }}=C\left(\frac{\nu_{s g s}}{\Delta}\right)^{2}
$$

with $C \approx 100$ (see references $[12,23]$ ). The eddy viscosity is known to theoretically vary as the filter size with a $4 / 3$ power law [24]. This allows finally to predict the new mesh size, $\Delta_{a}$, from the initial mesh size, $\Delta_{i}$, to respect this second criterion,

$$
\Delta_{a}=\Delta_{i}\left(\frac{0.2}{Q c_{2, i}}\right)^{3 / 2},
$$




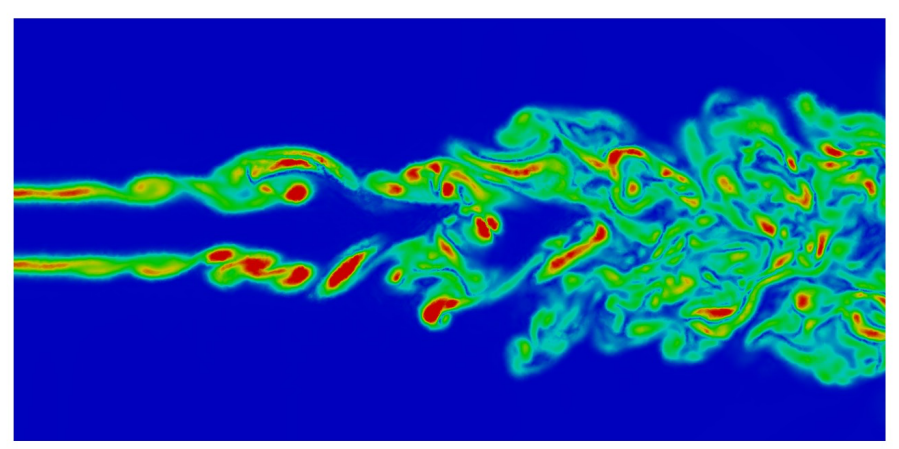

Figure 1. DNS of the turbulent plane jet: central plane colored by the vorticity magnitude.

where $Q c_{2, i}$ is the value of the second criterion computed on the initial mesh.

The proposed strategy to adapt mesh for LES specificity is then to evaluate $Q c_{1}$ and $Q c_{2}$ from an initial mesh and to define a new mesh by a minimization of $Q c_{1}$ and by using equation (5).

\subsection{Application in canonical test cases}

As an illustration, this strategy is now applied in the LES of canonical test cases: a turbulent plane jet and a turbulent pipe flow using the dynamic Smagorinsky model, as SGS model [25, 26]. For these test cases, the YALES2 flow solver [27, 28] is used. This code solves the low-Mach number NavierStokes equations for turbulent reactive flows on unstructured meshes using a projection method for constant [29] or variable density flows [30]. It relies on fourth-order central finite-volume schemes and on highly efficient linear solvers [31], which enable the simulation and the post-processing of iso-thermal or reacting flows on massive unstructured grids [32, 33].

As first test case, a turbulent plane jet is considered. The flow configuration is similar as the configuration studied by da Silva and Métais [34]. Thus, the inlet boundary condition is given by an analytic hyperbolic tangent profile with a white noise. The Reynolds number is $R e=$ $\Delta U H / \nu=3000$, with $H$ the initial thickness of the jet and $\Delta U=U_{j}-U_{c}$ with $U_{j}=1.091$ and $U_{c}=0.091$, the center jet and co-flow velocity, respectively. The computational domain size is $12.4 H \times 12 H \times 2.9 H$ in the streamwise, normal and spanwise directions. A DNS is first performed as reference. The DNS mesh is composed of 49.3 millions of tetrahedral cells with a roughly uniform 


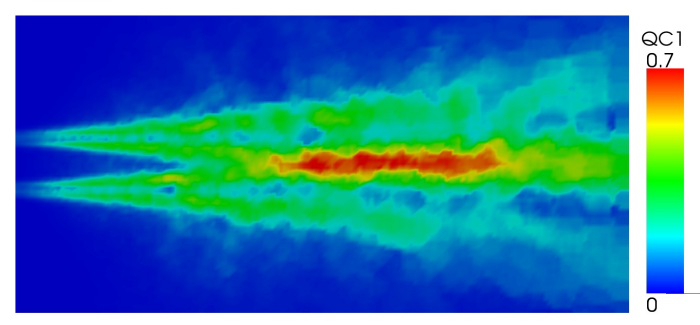

(a)

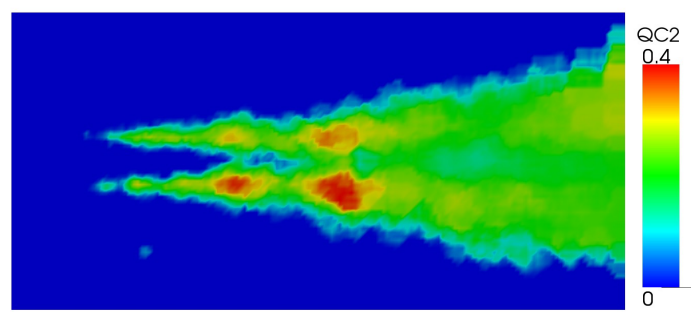

(b)

Figure 2. Central plane colored by $Q c_{1}$ (a) and $Q c_{2}$ (b) to evaluate the mesh quality of the initial mesh used in LES.

size (an edge size is around 0.04H, as in da Silva and Métais [34]). Figure 1 shows the jet dynamic. The first stages of the jet correspond to the development of the Kelvin-Helmholtz vortices. Then, a secondary instability grows, leading the jet through a fully developed turbulent state. A first LES computation is performed with the same numerical set-up, but with a coarse mesh only composed of 1.7 millions of tetrahedral cells, with edge sizes increasing from $0.04 H$ at the inlet, to $0.8 H$ at the outlet. The quantities $Q c_{1}$ and $Q c_{2}$ are then computed (Fig. 2). At the first transition stages, the major constraint for the mesh is due to the shear layers, where the mean velocity gradient is high. This is illustrated by the important value of $Q c_{1}$. In this region, there is no turbulent scales, explaining the small value of $Q c_{2}$. In the downstream region, the turbulent scales are developed according to the classic transition scenario in free shear flow. Then, $Q c_{2}$ grows first in the shear layers, and finally in all the turbulent jet after the end of the potential core. A second LES has been performed on an adapted mesh following the strategy described in the previous section. This new 


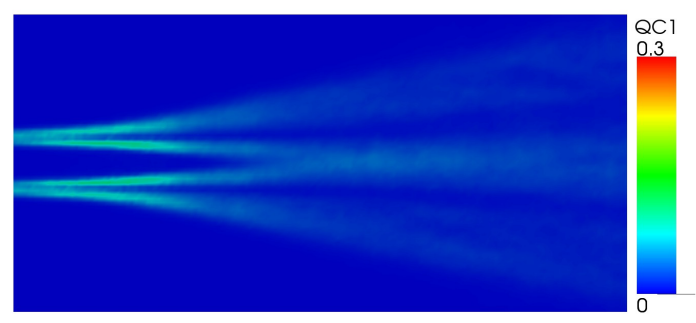

(a)

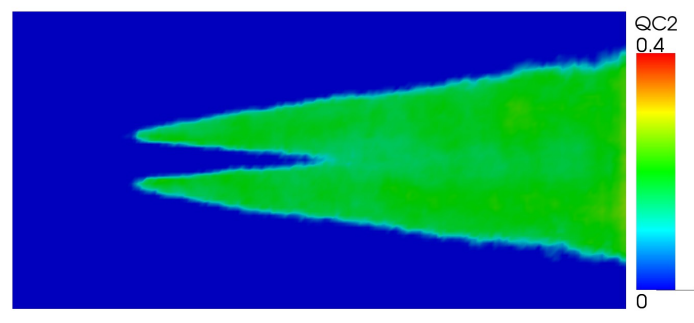

(b)

Figure 3. Central plane colored by $Q c_{1}$ (a) and $Q c_{2}$ (b) for the second LES mesh, defined from the initial mesh.

mesh is composed by 3.4 millions of tetrahedral cells with edge sizes increasing from $0.04 H$ to $0.3 H$. This new mesh allows to minimize $Q c_{1}$ and to respect $Q c_{2}<0.2$ as shown by Fig. 3. The mesh influence on the statistic prediction is shown by Fig. 4. First, Fig. 4 (a) compares the mean streamwise velocity profile at different sections. The initial LES mesh leads to some discrepencies with DNS results in the shear regions, due to an unsatisfactory discretization of the mean velocity gradient, whereas the second adapted LES mesh allows to correct this and leads to a good agreement with the DNS results. The disagreement between the DNS results and the results given by the initial LES mesh are more pronounced for the rms quantities, Fig. 4 (b)-(d). This is because the initial LES mesh does not allow to resolve enough turbulent scales as shown by the evaluation of $Q c_{2}$. The adapted LES mesh allows to correct that, and leads to a rms prediction in better agreement with DNS results. Note that, when rms quantities are considered, an exact agreement between DNS and LES results is not expected, because a part of the turbulent field is not resolved in LES. Vorticity 


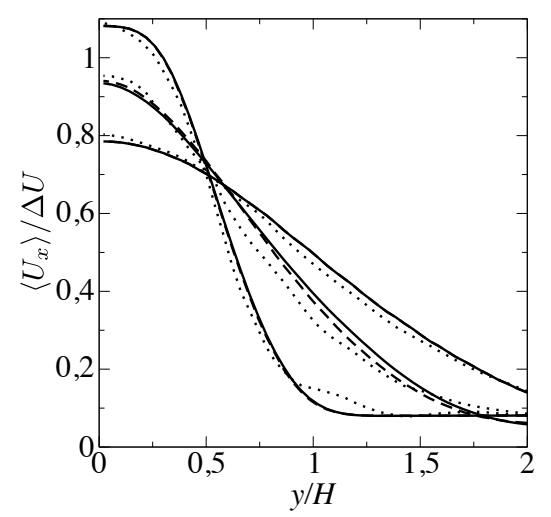

(a)

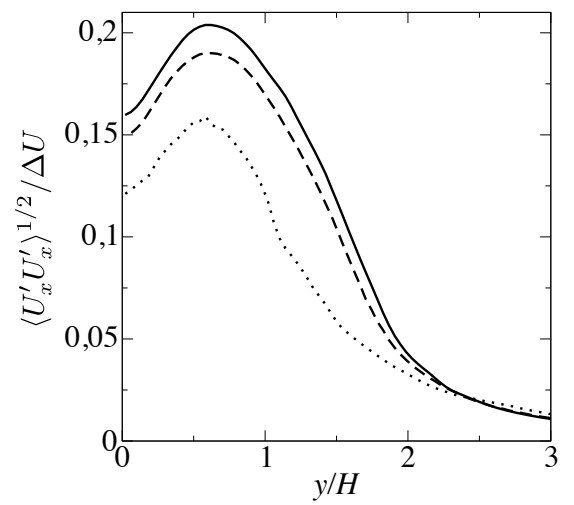

(c)

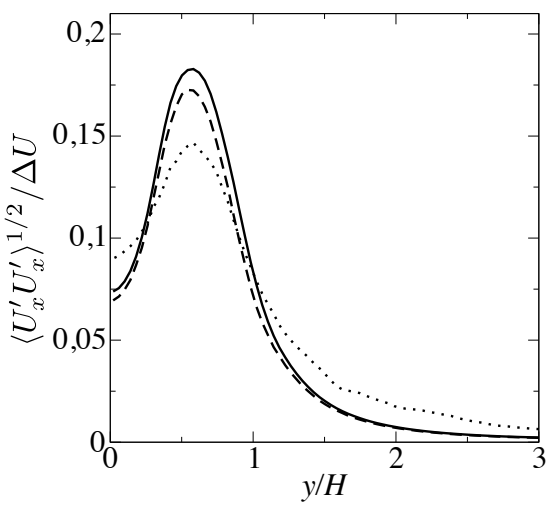

(b)

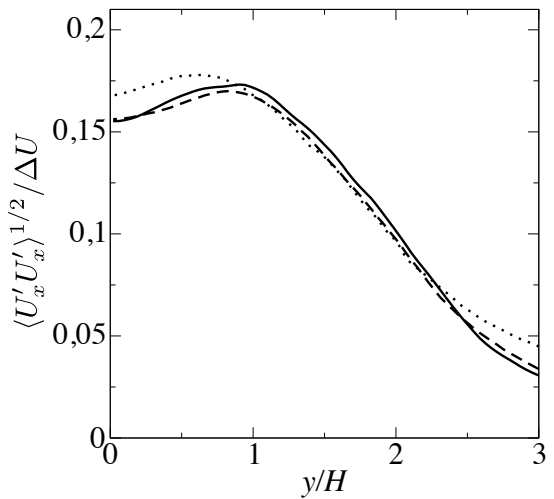

(d)

Figure 4. Comparison of turbulent plane jet statistics: DNS (solid line), first LES mesh (dotted line) and adapted LES mesh (dashed line). Mean axial velocity profile at three sections: $x / H=4,8$ and 12 (a) and rms velocity at $x / H=4(\mathrm{~b}), 8(\mathrm{c})$ and $12(\mathrm{~d})$.

fields obtained on the initial and on the adapted LES grids are represented in Fig. 5. The adapted mesh allows to recover more turbulent activity compared to the initial mesh but not all the activity shown in Fig. 1 as the small scales of turbulence are modeled. These first test cases allow to show the ability of the proposed strategy to deal with flow configuration in transition through a fully turbulent state.

The second test case deals with turbulent wall-bounded flow, by considering a turbulent pipe flow. The flow configuration is similar to a DNS performed by Wu and Moin [35] on a structured mesh composed of 67.7 millions cells. A periodic condition is used between the inlet and the outlet, with 


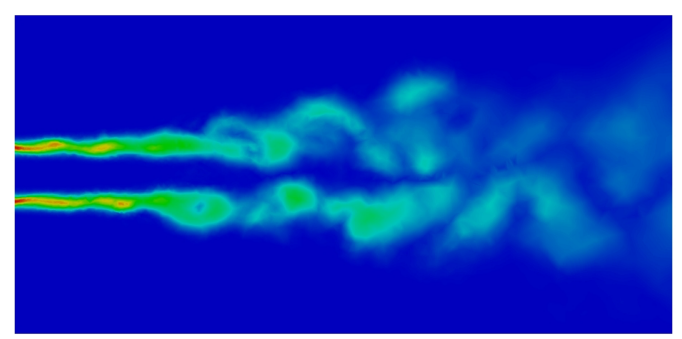

(a)

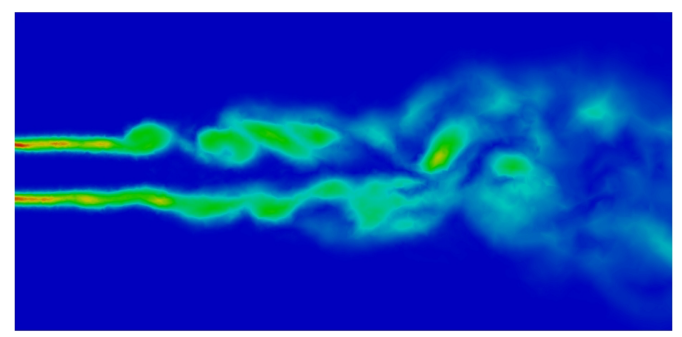

(b)

Figure 5. Central plane colored by the vorticity magnitude obtained with the initial LES mesh (a) and the adapted LES mesh (b).

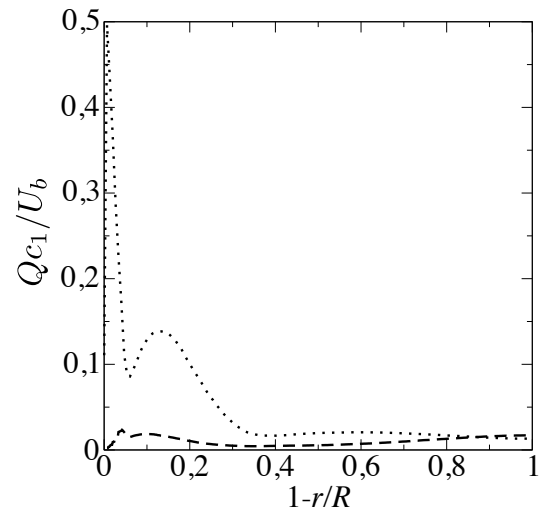

(a)

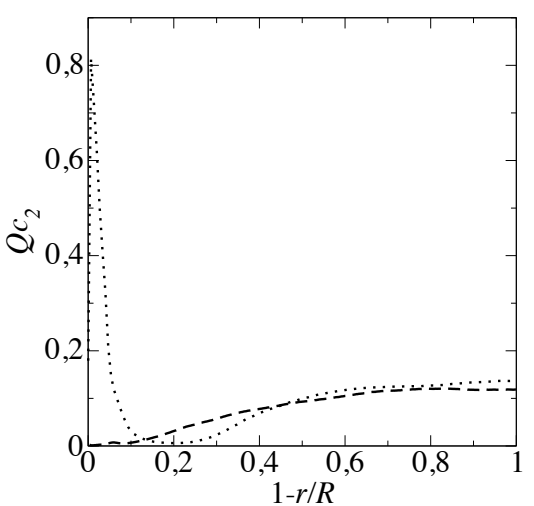

(b)

Figure 6. Profile of the quantities $Q c_{1} / U_{b}$ (a) and $Q c_{2}$ (b) for the initial LES mesh (dotted line) and for the adapted LES mesh (dashed line) for the turbulent pipe flow configuration. 


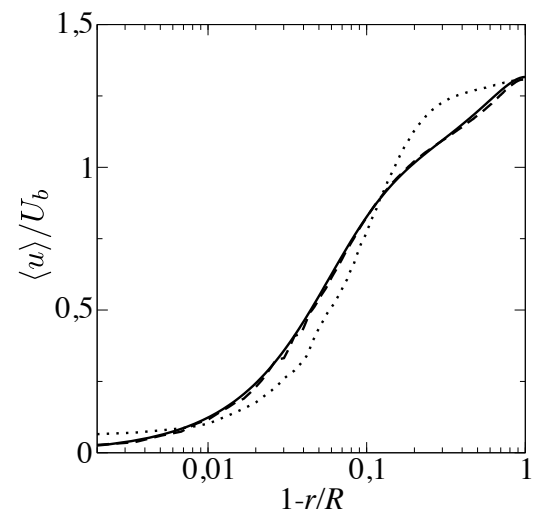

(a)

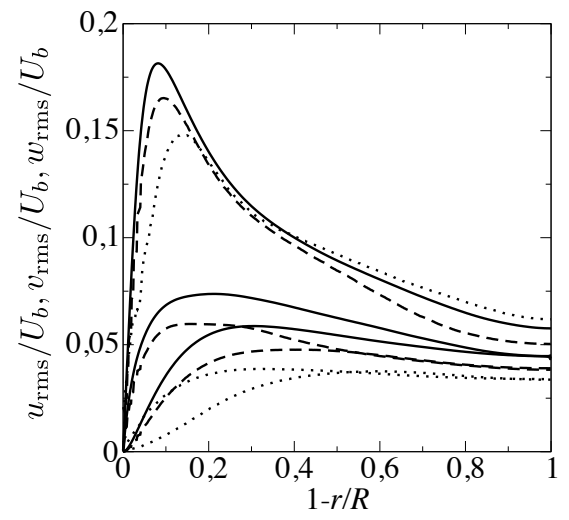

(b)

Figure 7. Mean velocity profile (a) and rms velocity profiles (b) for the turbulent pipe flow configuration: DNS (solid line), first LES mesh (dotted line) and adapted LES mesh (dashed line).

a forcing to ensure a constant bulk velocity $U_{b}$. The Reynolds number based on the bulk velocity and the pipe diameter is 5300. An initial LES is performed with a mesh composed of 1.1 millions tetrahedral cells with edge sizes increasing from $0.08 R$ at the wall, to $0.15 R$ at the center of the pipe, where $R$ is the pipe radius. The radial profile for quantities $Q c_{1}$ and $Q c_{2}$ are shown on Fig. 6. The value of these quantities are important close to the wall, showing that a refinement is needed in this region. This is well-known characteristic of turbulent wall-bounded flows, where the first cell close to the wall is expected to have a size around one wall unit [12]. For this initial LES mesh, the size of the wall cells is around 10 wall units. A second adapted LES mesh is then defined. This adapted LES mesh is now composed of 23 millions cells composed by prismatic cells close to the wall and tetrahedral cells in the center of the pipe. The edge sizes increase from $0.008 R$ to $0.12 R$. This new adapted LES mesh allows to respect both criteria, based on $Q c_{1}$ and $Q c_{2}$, as shown by Fig. 6. Figure 7 shows the mesh influence on statistic predictions, by comparison with DNS results of $\mathrm{Wu}$ and Moin [35]. As expected, the initial LES mesh is too coarse to allow a correct agreement with DNS results. This is due to an unsatisfactory discretization of the mean velocity gradient close to the wall. Moreover, the rms prediction are strongly under-estimate and the peaks of fluctuation are shifted to the core of the pipe. The adapted LES mesh allows to correct these behaviors. A 
very good agreement is found for the mean velocity profile and the locations of the rms peaks are better predicted. As already stated, note that rms predicted by LES is expected to be slightly underestimated in comparison with DNS, because the SGS part of the fluctuation is not known in LES.

\section{AUTOMATIC MESH ADAPTATION STRATEGY IN REALISTIC LARGE-EDDY}

\section{SIMULATIONS}

\subsection{Description of the parallel mesh adaptation algorithm}

Numerical simulations and parallel mesh adaptation are performed using the finite-volume LES code YALES2 $[27,28]$. The mesh adaptation module of YALES2 is based on the MMG3D library [36, 37]. MMG3D is a sequential anisotropic mesh adaptation library for tetrahedral elements based on local mesh modifications such as edge flips, edge collapsing, node relocations and vertex insertions driven by isotropic or anisotropic metric specifications.

In order to generate suitable meshes for LES problems, we develop a specific mesh optimization process driven by the improvement of the skewness [38]. The skewness of a tetrahedron $K$ is defined

as: $\frac{V_{\text {ref }}-V}{V_{\text {ref }}}$ where $V$ is the volume of $K$ and $V_{\text {ref }}$ is the volume of the equilateral tetrahedron included in the circumsphere at $K$. The optimization procedure is based on edge swaps and node relocations. The main difference with a standard mesh optimization process is the way to perform the node relocations. This procedure is devoted to improve the element in term of skewness and so we find a new position for the vertex such as the skewness of all the elements sharing this vertex increases.

The designed parallel mesh adaptation algorithm is an iterative process based on sequential calls to the MMG3D library on each processor. In order to minimize the rebuilding of mesh connectivity in parallel, which is computational expensive, the MMG3D library calls do not modify the surfaces formed of triangles that are shared by two processors or at the boundary of the computational domain. The triangles that are shared by two processors do not satisfy the metric specifications after an adaptation step. Thus, these triangles need to be moved to the interior of a processor in 
a subsequent step using a parallel graph partitioning algorithm. This parallel partitioning is also costly if performed at the cell connectivity level. In order to improve the performances, the parallel graph partitioning is conducted at the cell group level, each cell group containing approximately 500 elements.

Finally, given a grid, which is decomposed on a set of processors, the following procedure is carried out, illustrated by Fig. 8:

1. Sequential call to the MMG3D library on each processor leaving the boundaries on each processor untouched.

2. Splitting of the mesh on each processor to form cell groups.

3. Generation of a weighted connectivity graph between the cell groups with a strong weight on edges that are shared by two processors.

4. Parallel partitioning of the graph with an external library such as PARMETIS [39] or PTSCOTCH [40], which balances the cell groups per processor and minimizes the edge cut.

5. Rebuilding of the cell connectivity on each processor and in parallel.

6. Return to 1 . if not all the cells were treated.

Based on numerous numerical experiments, this iterative procedure converges in three to four steps. This algorithm has been successfully applied to the mesh adaptation of grids counting more than 40 million tetrahedral cells on 128 cores.

As boundary cells cannot be moved into the interior of a processor, they are not modified by the adaptation procedure. This is clearly a limitation of the proposed methodology.

\subsection{Cell count control for the interpolation error criterion $Q c_{1}$}

While the second criterion based on the amount of resolved turbulent kinetic energy is fully determined with no adjustable parameter, the first criterion based on the interpolation errors of the mean fields has an intrinsic degree of freedom, which is the error level. In realistic LES, this error level has to be homogeneous in the whole computational domain while keeping an acceptable cell 


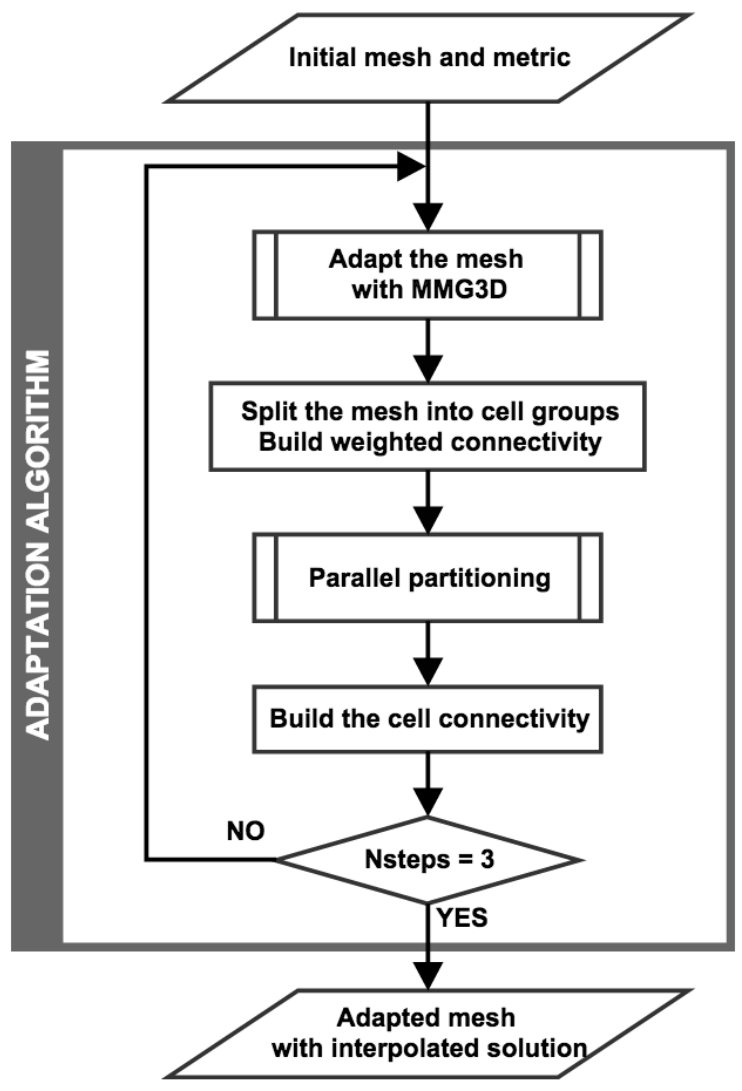

Figure 8. Algorithm of the parallel mesh adaptation.

count. It is therefore mandatory to propose an adaptation strategy based on the first criterion that allows the control of the cell count variation.

Assuming an isotropic mesh with cells of local size $\Delta$, the number of cells in a volume $V$ can be defined as the product of the local cell density $\rho$ and the volume itself. If the volume of a cell is approximately $\gamma \Delta^{3}$, where $\gamma$ is a constant, the number of cells in a given small volume $V$ is equal to $V /\left(\gamma \Delta^{3}\right)$. This leads to the following local cell count density $\rho=1 /\left(\gamma \Delta^{3}\right)$.

The total number of cells $N_{i}$ in a given initial computational domain $\mathcal{D}$ may be measured by the integral of the cell count density over the domain

$$
N_{i}=\int_{\mathcal{D}} \rho_{i} d V
$$

Defining a local refinement ratio $\tau$ as the cell size ratio between the initial and adapted meshes $\tau=\Delta_{i} / \Delta_{a}$, the previous equation enables to estimate the cell count of the adapted mesh from the 
initial cell density $\rho_{i}$ :

$$
N_{a}=\int_{\mathcal{D}} \rho_{a} d V=\int_{\mathcal{D}} \tau^{3} \rho_{i} d V
$$

Subsequently, the ratio of the cell counts in the adapted and initial meshes is then written as

$$
\begin{aligned}
\frac{N_{a}}{N_{i}} & =\left(\int_{\mathcal{D}} \tau^{3} \rho_{i} d V\right)\left(\int_{\mathcal{D}} \rho_{i} d V\right)^{-1} \\
& =\left(\int_{\mathcal{D}} \frac{\tau^{3}}{\Delta_{i}^{3}} d V\right)\left(\int_{\mathcal{D}} \frac{1}{\Delta_{i}^{3}} d V\right)^{-1} .
\end{aligned}
$$

Considering the first adaptation criterion, which aims at homogenizing the interpolation errors of the mean fields, the local refinement ratio may be written as $\tau=\sqrt{Q c_{1, i} / Q c_{1, a}}$ where $Q c_{1, i}$ and $Q c_{1, a}$ are the evaluations of the interpolation errors on the initial and adapted meshes, respectively. From Eq. (1), the cell count variation reads

$$
\frac{N_{a}}{N_{i}}=\left(\int_{\mathcal{D}} \frac{Q c_{1, i}^{3 / 2}}{\Delta_{i}^{3} Q c_{1, a}^{3 / 2}} d V\right)\left(\int_{\mathcal{D}} \frac{1}{\Delta_{i}^{3}} d V\right)^{-1} .
$$

The homogeneity of the interpolation errors is obtained when $Q c_{1, a}$ is constant over the full computational domain for a given cell count variation $N_{a} / N_{i}$. The previous equation enables to find this constant value:

$$
Q c_{1, a}=\left(\frac{N_{a}}{N_{i}}\right)^{-\frac{2}{3}}\left(\int_{\mathcal{D}} \frac{Q c_{1, i}^{3 / 2}}{\Delta_{i}^{3}} d V\right)^{\frac{2}{3}}\left(\int_{\mathcal{D}} \frac{1}{\Delta_{i}^{3}} d V\right)^{-\frac{2}{3}}
$$

To summarize, the homogenization of the interpolation errors over the computational domain while controlling the variation of the total cell count of the mesh, may be performed in five steps:

1. Evaluation of the $Q c_{1, i}$ criterion from Eq. (1).

2. Computation of $Q c_{1, a}$ for a given cell count ratio $N_{a} / N_{i}$ from Eq. (11).

3. Calculation of the local mesh refinement ratio $\tau=\left(Q c_{1, i} / Q c_{1, a}\right)^{1 / 2}$.

4. Modification of the local mesh refinement ratio to cope with additional constraints: imposed minimum and maximum cell size, limitation of the cell size gradient, ...

5. Adapt the mesh.

Step 4 is an iterative procedure. Indeed, while an imposed minimum or maximum cell size or a limit of the cell size gradient are local constraints, the control of the total cell count of the final mesh 


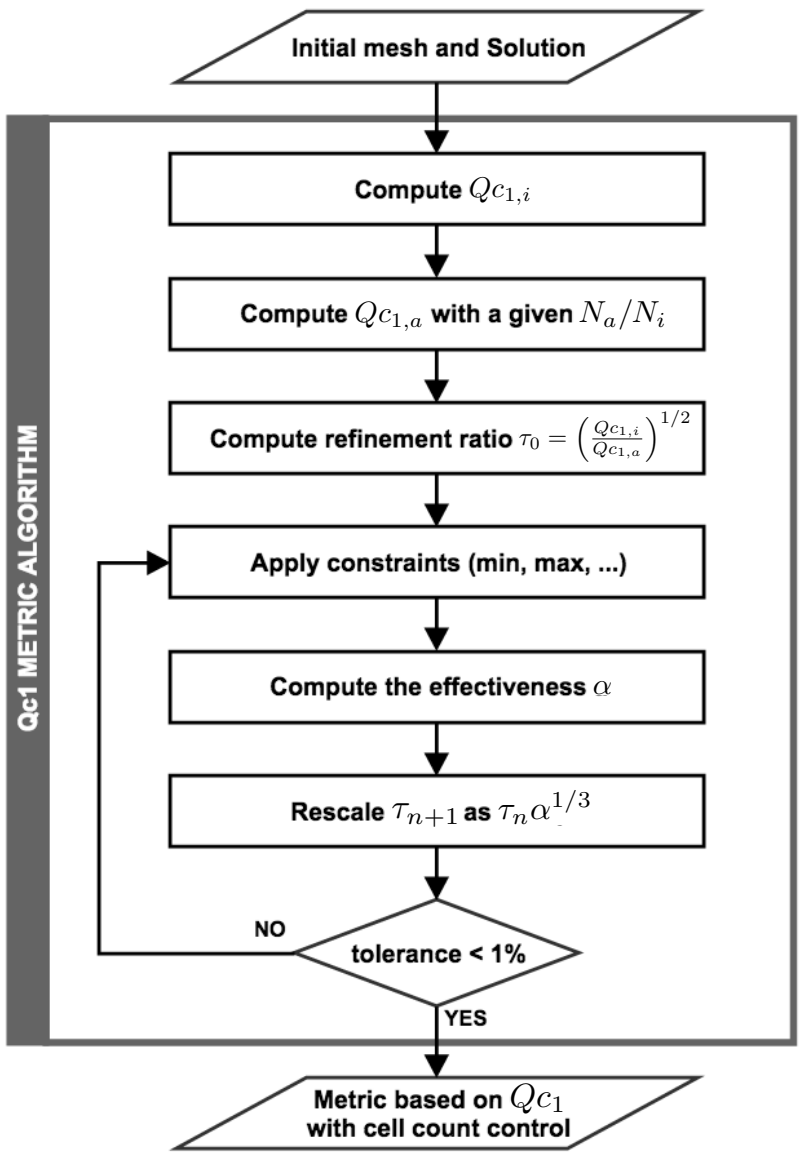

Figure 9. $Q c_{1}$ metric computation procedure with cell count control.

is a global constraint. The mesh refinement ratio needs to be adjusted over the whole computational domain to satisfy all the constraints. Consequently, a rescaling coefficient $\alpha$ obtained from Eq. 9 is introduced and determined iteratively:

$$
\alpha=\left(\frac{N_{a}}{N_{i}}\right)^{-1}\left(\int_{\mathcal{D}} \frac{\tau^{3}}{\Delta_{i}^{3}} d V\right)\left(\int_{\mathcal{D}} \frac{1}{\Delta_{i}^{3}} d V\right)^{-1} .
$$

The coefficient $\alpha$ is related to the effective refinement ratio $\tau_{n+1}$ as $\alpha=\left(\tau_{n} / \tau_{n+1}\right)^{3}$. The iterative procedure enables the coefficient $\alpha$ converging towards unity. An error tolerance of $1 \%$ on the cell count is imposed in this paper. 


\subsection{Adaptation strategy in realistic LES}

For LES of realistic cases, the following global strategy is proposed. The objective of this strategy is to define an optimal mesh for LES computations, i.e. respecting both criteria with a minimum number of cells.

An iterative adaptation process is proposed here to produce new LES meshes. It can be described as follows:

1. Computations are performed on a mesh and statistics are collected,

2. $Q c_{1}$ adaptation criterion is computed from the time-average flow velocity,

3. A metric is derived from the criterion with cell number conservation and by adding new constraints (procedure of section 3.2),

4. An adapted grid is generated with this target metric by calling the MMG3D library (methodology of section 3.1),

5. Reset the statistics and go back to step 1 .

This process permits to have a smooth evolution of the grid between two process iterations and ensures the velocity gradients to be fully captured. The obtained grid is called A1. The criterion $Q c_{2}$ is then applied on A1 with no restriction on the number of tetrahedrons and by taking the more restrictive local cell size between the A1 grid and the metric obtained from $Q c_{2}$, resulting in a second grid A2. All this procedure is illustrated by Fig. 10. Applying the criteria in the given order will lead to a mesh with a concentration of cells in the mean flow gradient areas and where turbulent kinetic energy is important. With the inverse order, the resolution level of the turbulent kinetic energy would not be guaranteed as the $Q c_{1}$ metric algorithm can change the metric obtained from $Q c_{2}$.

As the $Q c_{1}$ criterion targets the mean velocity resolution by homogenizing the truncation errors, it affects directly the wall normal resolution and so the wall unit value. The wall resolution in other directions is not changed by the adaptation procedure as the surface mesh is not modified. 


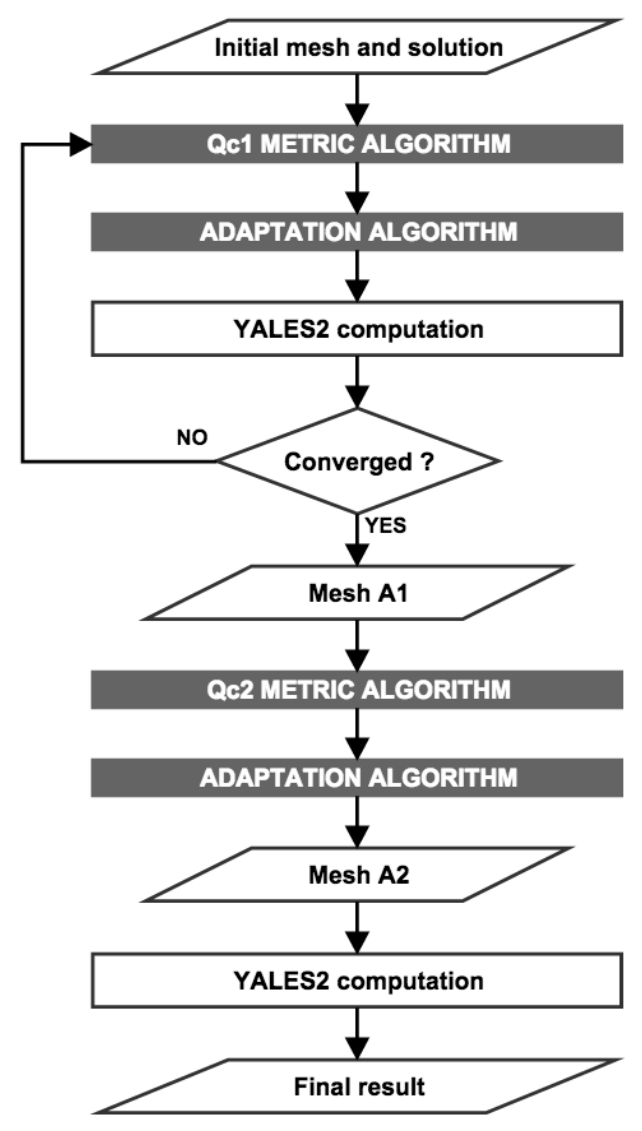

Figure 10. Local mesh adaptation procedure with $Q c_{1}$ and $Q c_{2}$ criteria.

It is important to note that the $Q c_{2}$ criterion is only valid for homogeneous isotropic turbulence but has been tested with success on numerous non-homogeneous or non-isotropic flows [8, 41]. The association of both criteria allows the handling of numerical and modeling errors.

\section{APPLICATION TO THE SIMULATION OF THE ISO-THERMAL FLOW IN A}

\section{MESO-SCALE COMBUSTOR}

\subsection{Description of the meso-scale device}

The investigated device is the meso-scale combustor studied experimentally in Liu et al. [42]. As shown in Fig. 11, the fluid volume is basically a cuboid which dimensions are $8 \times 8 \times 10 \mathrm{~mm}^{3}$. The 


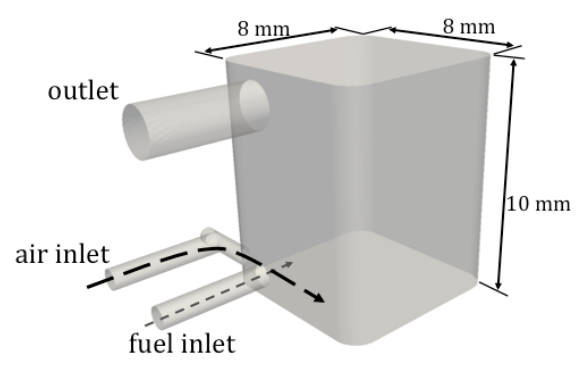

(a)

(b)

Figure 11. Three-dimensional modeling of the studied mesocombustor (a) and schematic of the computed fluid volume (b).

\begin{tabular}{lc}
\hline Inlet & Mean velocity $\left[\mathrm{m} . \mathrm{s}^{-1}\right]$ \\
\hline "Air" & 23.68 \\
"Fuel" & 1.20 \\
\hline
\end{tabular}

Table I. Operating conditions for the considered computation.

chamber is fed by two inlets: one for fuel, with a $1 \mathrm{~mm}$ diameter, another for air, with a $0.8 \mathrm{~mm}$ diameter. The outlet diameter is $2 \mathrm{~mm}$.

A reference computation of the fluid flow is performed with a 273 million tetrahedrons mesh called MREF. This mesh was built from an original grid called M1, created with a commercial software, composed of 4.5 million tetrahedrons with an homogeneous cell size of $94 \mu \mathrm{m}$. Then, two levels of homogeneous mesh refinement were performed starting from M1. At each refinement level, the cells are divided in eight subcells and the cell size is divided by two [43]. The resulting grid MREF has a homogeneous cell size of $24 \mu \mathrm{m}$.

Non-reactive computations are performed using the YALES2 incompressible solver. Thus, a unique gas, with a constant density of $\rho=1 \mathrm{~kg} \cdot \mathrm{m}^{-3}$ and constant kinematic viscosity $\nu=$ $1.517 \times 10^{-5} \mathrm{~m}^{2} \cdot \mathrm{s}^{-1}$, is used here. The operating conditions are summarized in Table I. 


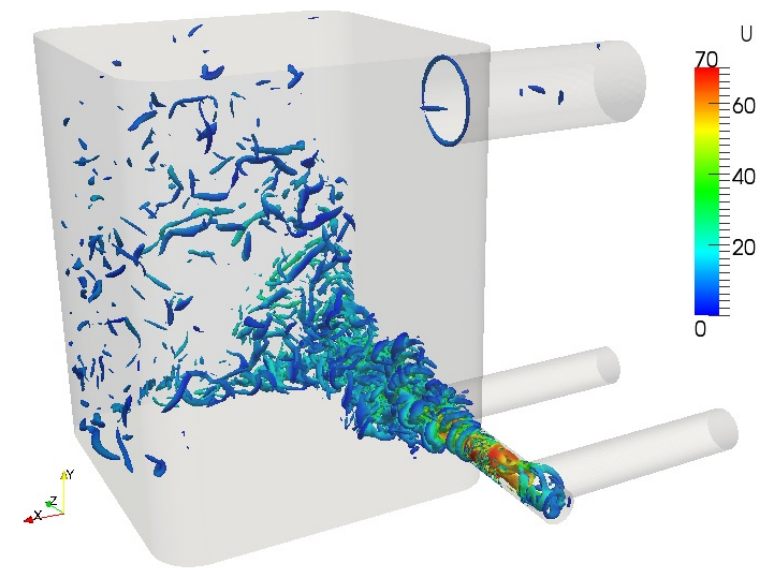

(a)

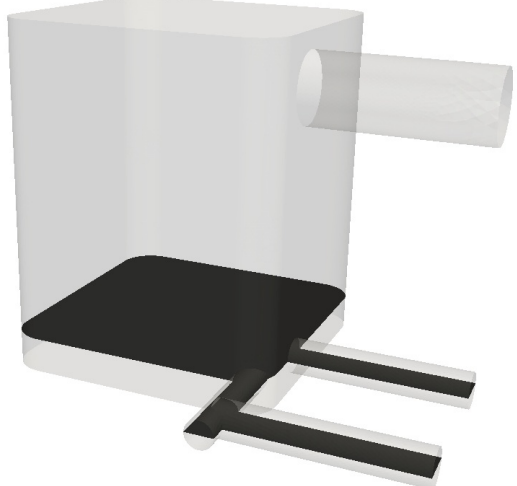

(b)

Figure 12. Iso-contour of Q-criterion $Q=1 \times 10^{9} \mathrm{~s}^{-2}$ colored by the velocity (a) and visualization of the injection plane (in black) in a 3D view (b).

The turbulent viscosity WALE model [44] was used in this paper. Preliminary studies showed that it is the most appropriate model in our configuration [45].

The feeding system is complex as the air flow enters the combustor tangentially to the wall, while the fuel enters perpendicularly to the air jet. As previous experimental work [42] and simplified DNS modeling [46] showed, the air jet impinges the upward wall, then deflects to create a very strong vortex at the center of the combustion chamber [47]. The coherent structures of the turbulent flow can be observed by means of the Q-criterion [48] computed as $Q=\frac{1}{2}\left(\Omega_{i j} \Omega_{i j}-S_{i j} S_{i j}\right)$ with $\Omega_{i j}=\frac{1}{2}\left(\partial_{j} u_{i}-\partial_{i} u_{j}\right)$ and $S_{i j}=\frac{1}{2}\left(\partial_{j} u_{i}+\partial_{i} u_{j}\right)$. As shown on Fig. 12 (a), production of turbulent structures can be observed in the air inlet pipe when the flow is deviated by an elbow before entering the chamber, in the jet shear layers zones and at the impact of the impinging air jet on the opposite wall. It appears that the injection area play a major role on the rest of the flow. It is also here that can be found the higher velocities and velocity gradients. As the most interesting part of the flow, the results that follow will be compared thanks to the injection plane showed in Fig. 12 (b). 


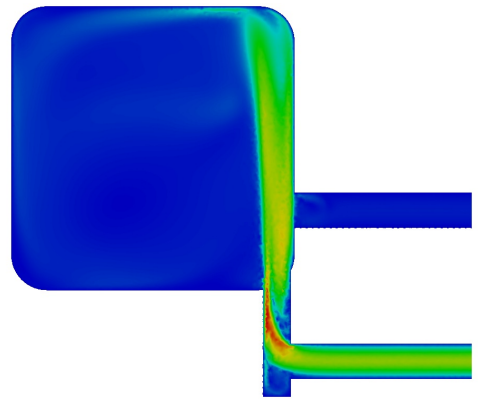

(a)

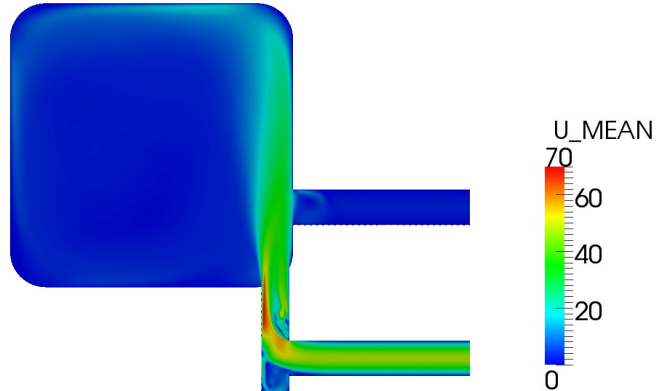

(b)

Figure 13. Injection plane mean velocity field on the M1 (a) and MREF (b) meshes in m/s.

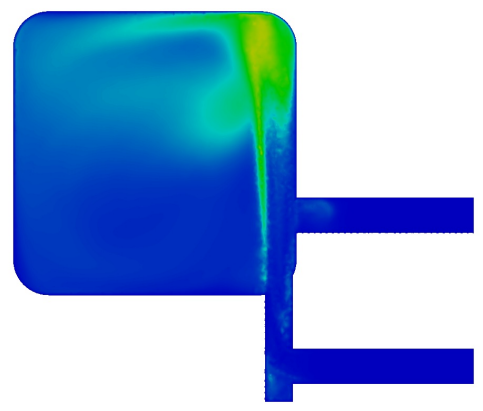

(a)

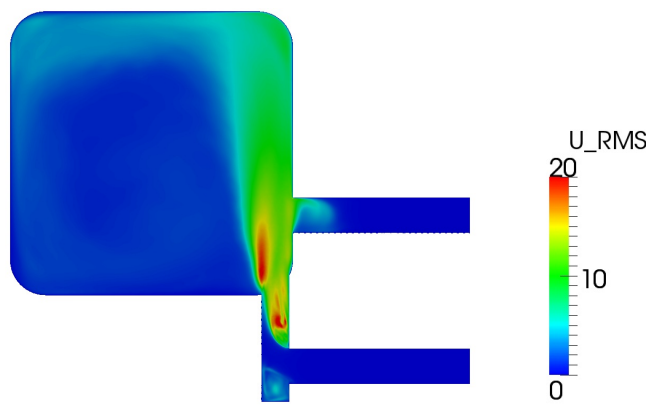

(b)

Figure 14. Injection plane rms velocity field on the M1 (a) and MREF (b) meshes in m/s.

\subsection{Homogeneous mesh refinement study}

Computations is performed with the M1 and MREF grids. Figure 13 shows the mean velocity fields on the injection plane. On these two meshes the shape and angle of the air jet is different between the coarse and the fine grids. Furthermore, it can be observed that the rest of the flow is dependent on the jet description and on the creation of the turbulent structures.

Figure 14 shows the rms velocity fields on the injection plane for the two meshes. Their behavior are completely different. On the beginning of the air jet in the combustor, MREF shows high velocity fluctuations while there are not on M1. On M1, fluctuations can be observed on all the shear stress layer between the air jet and the rest of the combustor when MREF presents velocity fluctuations on all the air jet. At the impact of the jet on the wall, rms velocity values are lower on the refined 
grid. The mean wall unit value is around 2 on the M1 grid and lower than 1 on MREF. This allow the computation to be sufficiently resolved at the walls for both cases.

As the cell size is an input for LES sub-grid scale models, it can have a direct influence on the turbulent viscosity and then on the overall result of an LES computation. As a reminder, the mean cell size of MREF, around $24 \mu \mathrm{m}$, is so small that the turbulent viscosity in the domain by the SGS model is negligible. The MREF computation is assumed as a DNS case.

All these observations result in flow dynamics differences between the two computations. It is expected that an LES adapted mesh allow a better description of the velocity field compared to M1, but with less nodes as needed with MREF.

\subsection{Local mesh refinement study}

The parallel mesh adaptation procedure described in 3.1 was applied to the meso-scale configuration, starting from M1 mesh and with the help of mesh criteria of section 2.1. Statistics are collected on $200 \mathrm{~ms}$ which corresponds to ten times the mean residence time. The obtained metric is clipped between 20 and $500 \mu \mathrm{m}$ in order to avoid too small or too large cells.

Figure 15 shows the criteria $Q c_{1}$ and $Q c_{2}$ obtained on the A1 and $\mathrm{A} 2$ grids. On the $\mathrm{A} 1 \mathrm{mesh}$, the $Q c_{1}$ field is homogeneous except at the walls where cells are not adapted. So the interpolation error is well distributed in all the domain. However, the $Q c_{2}$ is higher than 0.2 in a large part of the cutting plane. Turbulent kinetic energy is largely modeled here. The A2 grid show lower values of $Q c_{1}$ than A1 at the centre of the domain. As it contains more elements, the interpolation error is even lower. On the other hand, the $Q c_{2}$ criterion is mostly below 0.3 in the domain, so largely reduced from A1. High $Q c_{2}$ values can be found in the inlet pipes but these areas show very low turbulent kinetic energy so the SGS model has no impact.

The overall grid statistics are summarized in Tab. II. The adapted grid A1 shows a number of elements close to the original mesh M1 while the one adapted from $Q c_{2}$ exceeds 14 millions elements. As presented on Fig. 16, the distribution of the M1 grid is homogeneous and centered around $94 \mu \mathrm{m}$, while the distribution for MREF follow the one of M1 but with four times smaller 


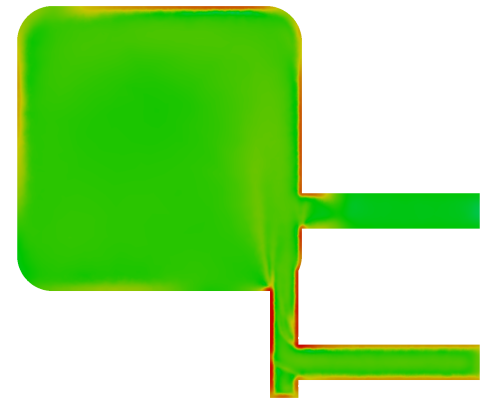

(a)

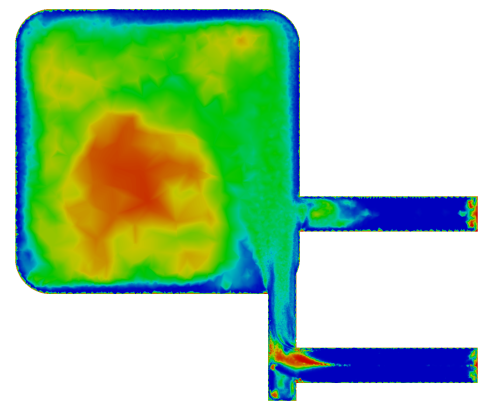

(c)

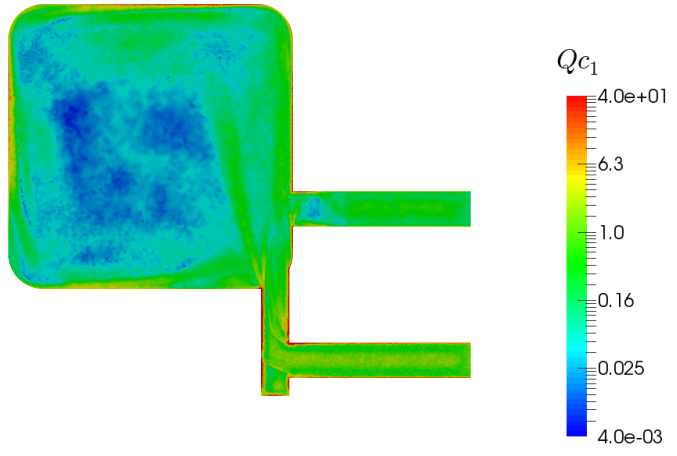

(b)

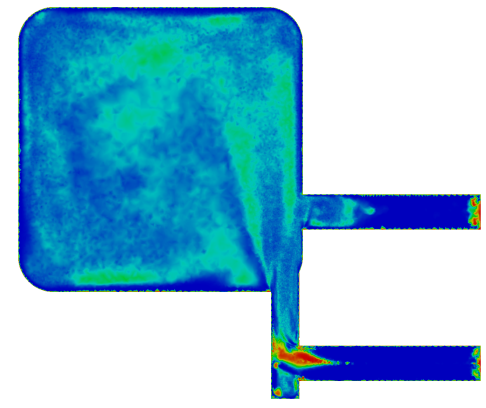

$Q c_{2}$

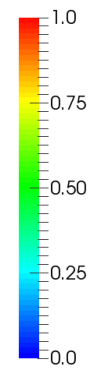

(d)

Figure 15. $Q c_{1}$ (top) and $Q c_{2}$ (bottom) criteria fields on injection cutting plane on A1 (left) and A2 (right) meshes.

\begin{tabular}{lcccc}
\hline & M1 & MREF & A1 & A2 \\
\hline Elements & $4^{\prime} 483^{\prime} 330$ & $273^{\prime} 751^{\prime}, 808$ & $4^{\prime} 335^{\prime} 692$ & $14^{\prime} 808^{\prime} 033$ \\
Nodes & $79^{\prime} 388$ & $47^{\prime} 985^{\prime} 812$ & $757^{\prime} 779$ & $2^{\prime}{ }^{\prime} 504^{\prime} 555$ \\
Mean cell size $[\mu \mathrm{m}]$ & 94 & 24 & 95 & 64 \\
Time step $[\mu \mathrm{s}]$ & 1.77 & 0.379 & 0.269 & 0.255 \\
Normalized CPU cost & 1.0 & 358.5 & 16.0 & 92.9 \\
\hline
\end{tabular}

Table II. Characteristics of the studied grids.

values of cell size. Starting at $20 \mu \mathrm{m}$, the minimal cell size target, the cell size distribution of A1 and $\mathrm{A} 2$ are more heterogeneous. 


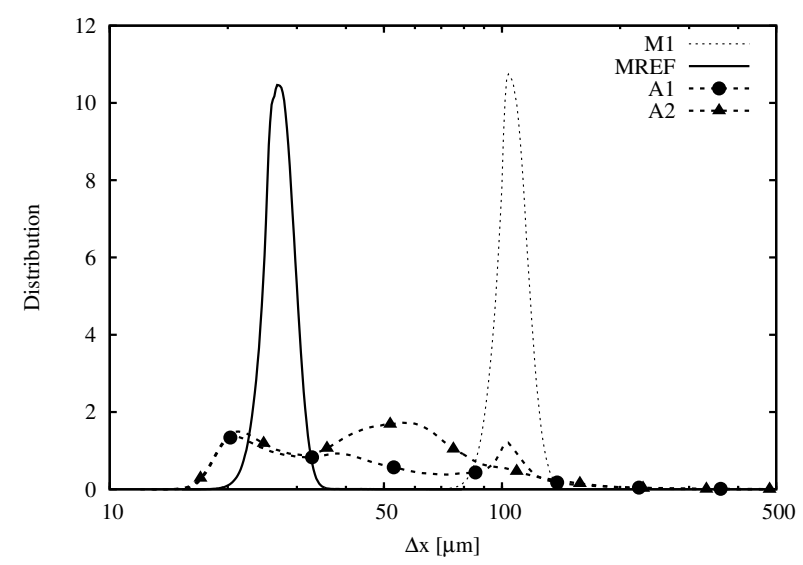

Figure 16. Cell size distribution of the M1, MREF, A1 and A2 grids.

This heterogeneous distribution is observed on Fig. 17 on the injection cutting plane. Compared to the M1 grid, the A1 mesh mainly adds nodes in the air inlet pipe, at the beginning of the jet and close to the walls. These zones corresponds to the higher mean velocity gradients areas showed by Fig. 13 (b). The mesh is less dense in the rest of the domain. The A2 grid show a high node density in the air inlet pipe and in the jet area. Smaller cells are also observed near the walls. The mesh density is globally higher for the A2 mesh since it contains three times more nodes than M1 and A1 grids.

Summarized in Tab. II, the CPU cost is computed as the time needed to compute the $200 \mathrm{~ms}$ physical time used for statistics. It is then normalized by taking CPU cost of M1, which is 6.7 hours on 128 Intel Nehalem processors. The two adapted grids computations are 16.0 to 92.9 more expensive than with M1 but are still 22 to 4 times cheaper than the automatically refined mesh MREF. Although the grid A1 shows a similar number of cells to M1, it contains a minimal cell size smaller than the smallest of grid M1. Thus, the fluid solver time step is substantially affected.

The mean velocity field obtained with the adapted grids, presented on Fig. 18, seem to be very similar to each other and very close to the reference computation of Fig. 13 (b). On the other hand, they vary considerably from the M1 computation.

Presented on Fig. 18, rms velocity fields on the adapted meshes show a huge improvement compared to the result on the M1 grid (Fig. 13 (a)) and are very similar to the reference. Both 


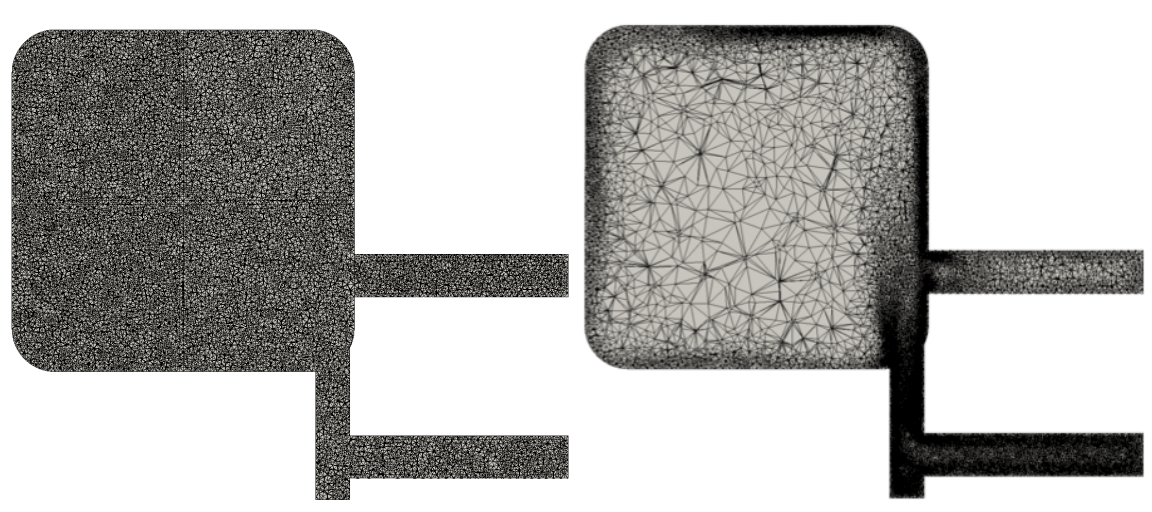

(a)

(b)

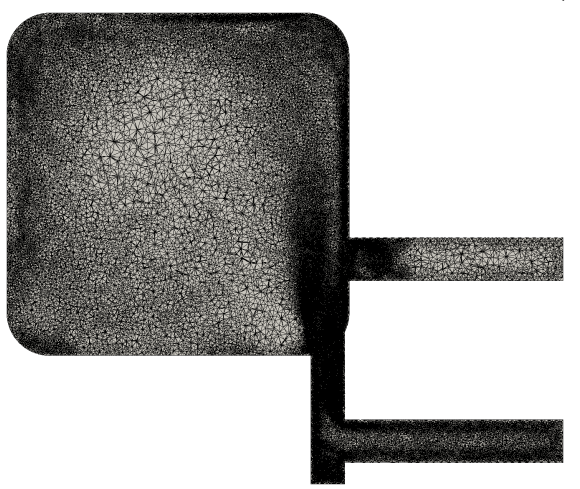

(c)

Figure 17. Comparison of the M1 (a), A1 (b) and A2 (c) grids in the injection plane.

A1 and A2 meshes present high rms values on the jet, just after the geometry elbow. At the jet impact, lower values of rms velocity are observed on the A1 grid compared to A2 and MREF. A too poor resolution in this area can explain this discrepancy.

Quantitative comparisons of velocity profiles are performed on four lines into the combustor shown by Fig. 19. Fig. 20 presents the mean velocity profiles on these lines compared to the reference. The profiles obtained from the M1 mesh present many discrepancies compared to the other meshes. However, the comparison between the adapted and the reference meshes is not quantitative. To quantify the deviation from the reference, L2 norm error of mean velocity profiles are also computed. The Tab. III confirms that the M1 solution is far from the reference: the error is 


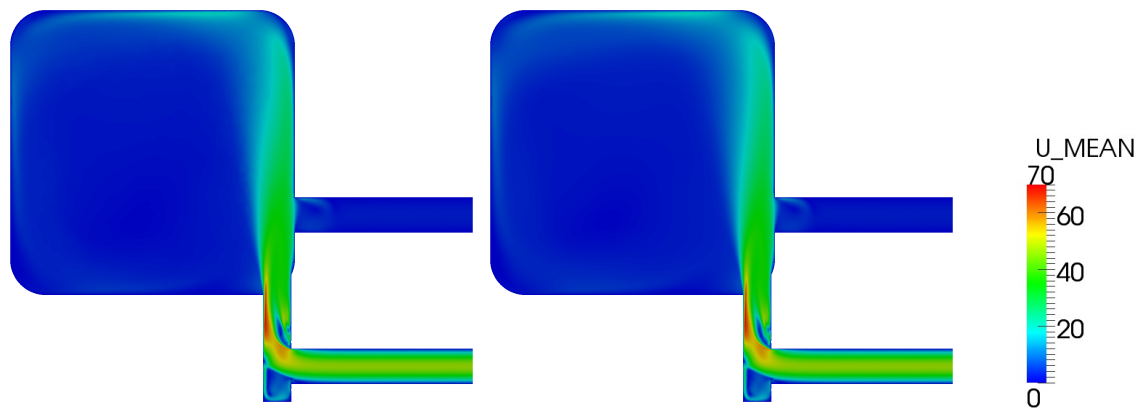

(a)

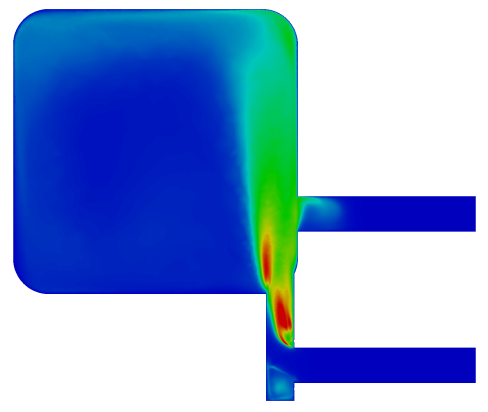

(c) (b)

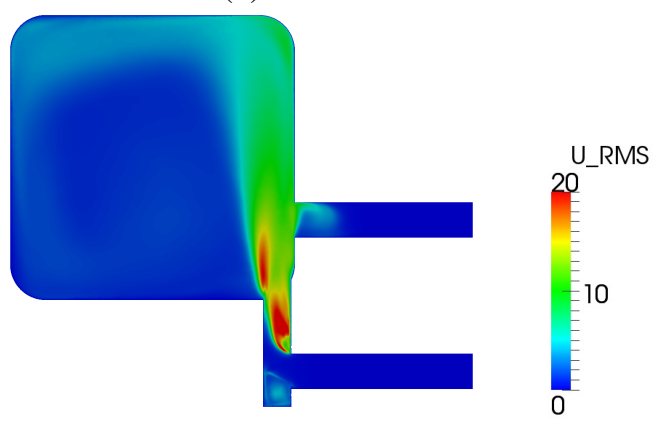

(d)

Figure 18. Mean (top) and rms (bottom) velocity fields on the A1 (left) and A2 (right) meshes.

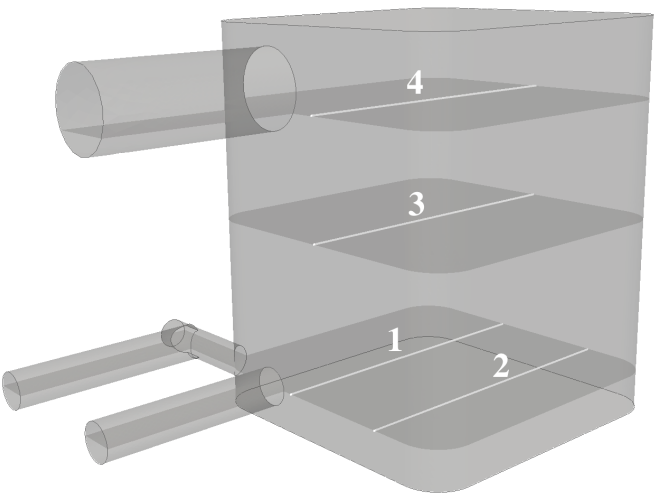

Figure 19. Visualisation of the lines 1,2, 3 and 4.

between 13 and $60 \%$. The solution based on the mesh A1 improves this result but the A2 grid shows the best agreement with the reference: the relative L2 norm error is always under $7 \%$.

Fluctuation velocity profiles are plotted on Fig. 21 on the four lines. Profiles from A2 grid always show a good agreement with the reference. At the opposite, worst results can be observed from the 


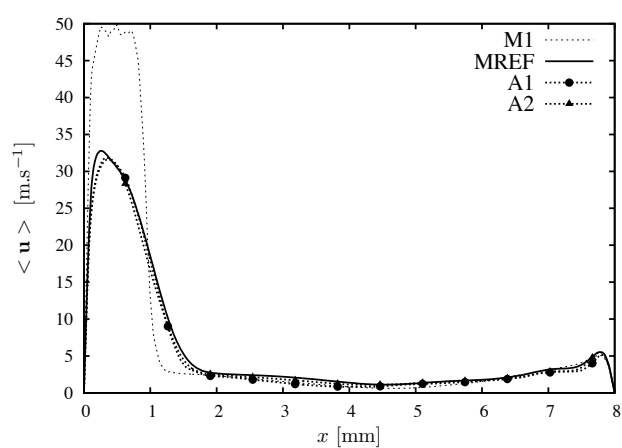

(a)

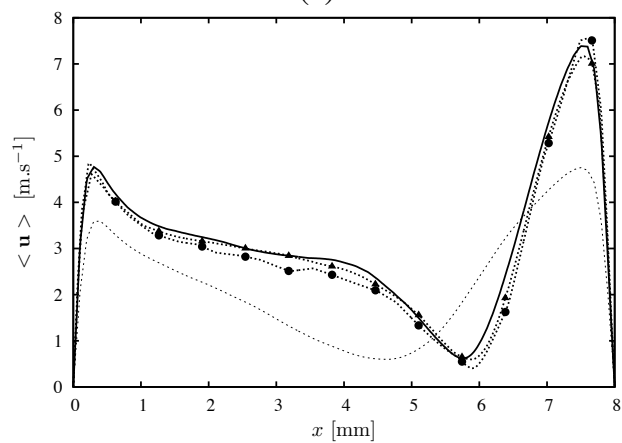

(c)

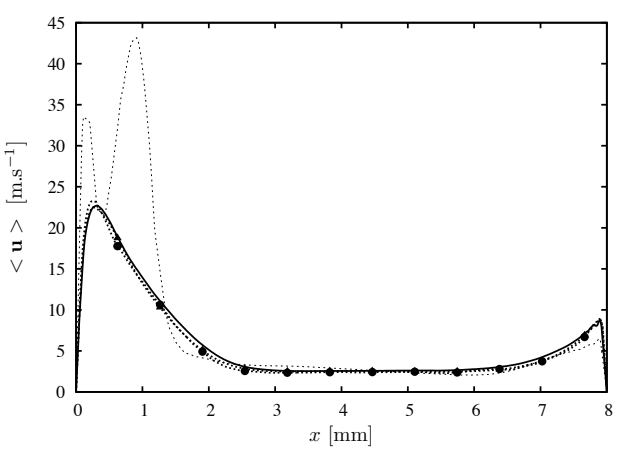

(b)

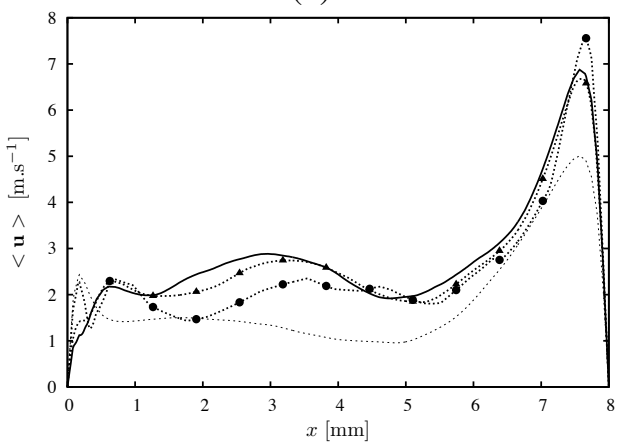

(d)

Figure 20. Mean velocity profiles on lines 1 (a), 2 (b), 3 (c) and 4 (d).

\begin{tabular}{lccc}
\hline Lines & M1 & A1 & A2 \\
\hline 1 & 60.1 & 5.4 & 4.5 \\
2 & 30.0 & 4.7 & 3.4 \\
3 & 13.6 & 7.3 & 4.0 \\
4 & 18.4 & 14.7 & 6.5 \\
\hline
\end{tabular}

Table III. Relative L2 norm error of mean velocity profiles of four lines and on the different meshes in $\%$.

A1 grid, due to lack of resolution away from the injection area. This show the superiority of the A2 grid and the influence of the sub grid scale modeling impact. It confirms the choice of the two adaptation criteria. 


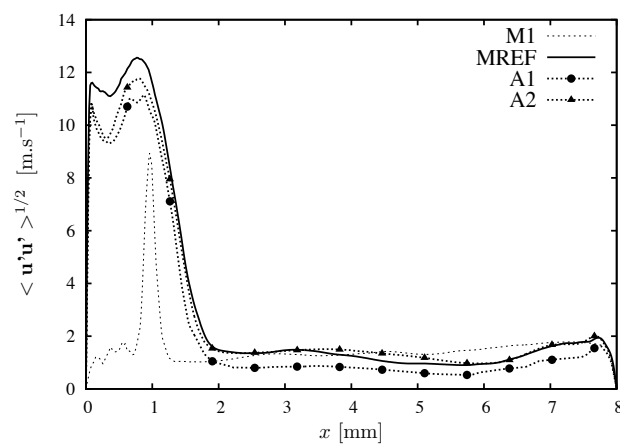

(a)

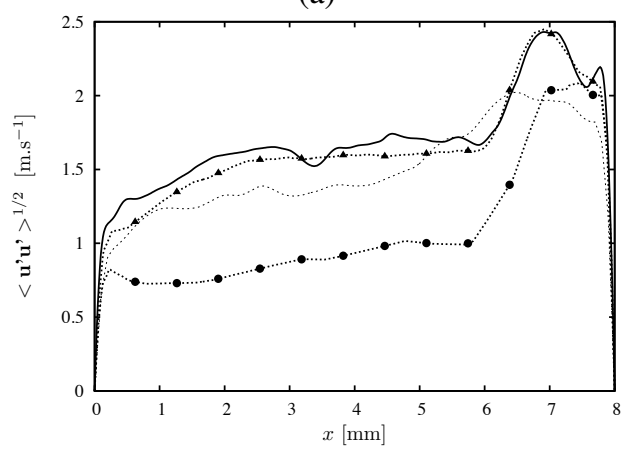

(c)

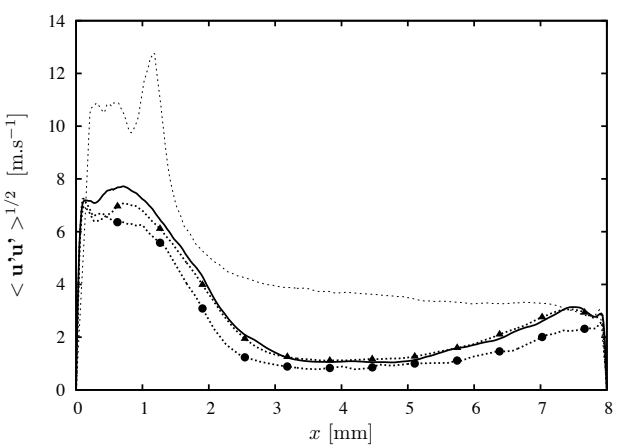

(b)

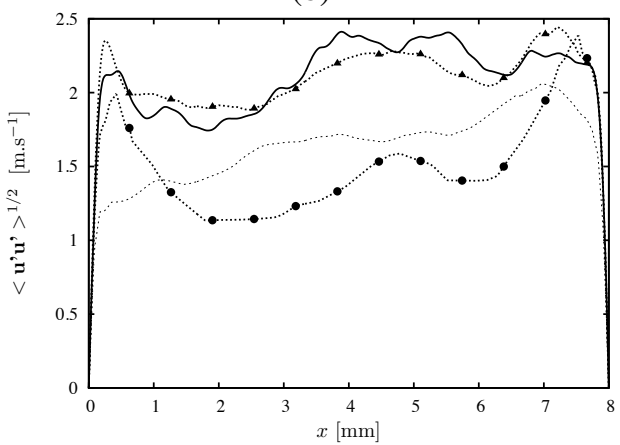

(d)

Figure 21. Fluctuation velocity profiles on lines 1 (a), 2 (b), 3 (c) and 4 (d).

\section{CONCLUSION}

This paper presents a local mesh adaptation methodology for LES of turbulent flows in complex geometries. This work contributes to addressing the grid dependence issue, which is inherent to implicit filtering LES. This methodology is based on two different mesh quality criteria, which target the discretization errors of the mean flow field and the resolution of a sufficient part of the turbulent spectrum, respectively. These criteria are combined to an efficient and parallel mesh adaptation algorithm built upon the MMG3D library. This latter is a sequential anisotropic adaptation library, which features mesh quality improvement filters. The adaptation methodology has been applied to the turbulent flow inside a centimeter-scale combustor with a complex geometry. The major results of this study may be summarized as follows 
- Mesh adaptation improves largely the quality of the results with a moderate over-cost. This strategy is far more efficient than brute force homogeneous refinement.

- With the first criterion, which aims at decreasing the discretization errors of the mean flow field, the mean and rms velocities are improved even if the total number of cells is kept constant. Compared to the original simulation, the calculation with the adapted mesh is more costly due to small cells, which have a more stringent time step restriction.

- With the second criterion, based on the resolution of $80 \%$ of the turbulent kinetic energy, the rms velocity is better predicted than with the first criterion at the expense of an increased mesh size and an additional cost.

- The numerical experiments performed on the meso-combustor also shown that the multi-step refinement procedure for a given criterion converges towards an adapted mesh.

The present methodology can be further improved to target larger meshes or enable surface refinement, which was not possible with the former versions of the MMG3D library. The paper investigates only two basic refinement criteria but many other criteria can be developed based on the physics of the flow. Finally, as the multi-step refinement procedure converges to an adapted mesh, it would be interesting to check if the obtained meshes are independent of the initial mesh. This would confirm that mesh-independent LES is tractable.

\section{APPENDIX}

Figure 22 presents the intermediate adapted meshes obtained during the $Q c_{1}$ adaptation algorithm. Starting from the M1 mesh (Fig. 17 (a)), the grid converges towards a unique solution: elements are added into the inlet pipes and in the jet area. In order to keep a constant number of cells, some elements are removed to the centre of the domain where velocity gradients are weak. The obtained converged grid A1 is shown in Fig. 17 (b). 


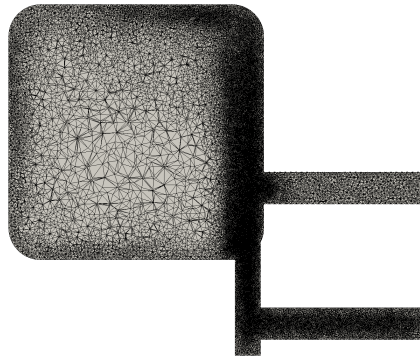

(a)

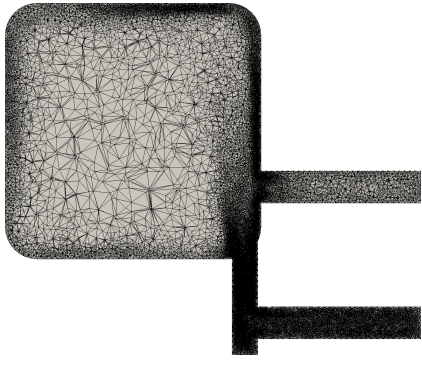

(b)

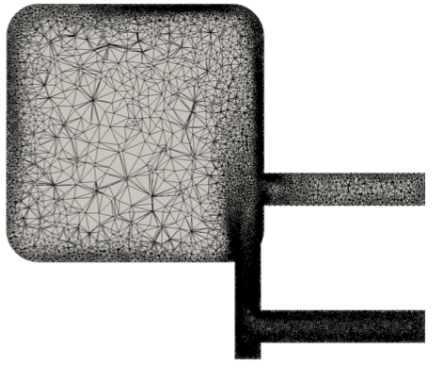

(c)

Figure 22. First (a), second (b) and final (c) grids obtained during the adaptation process based on $Q c_{1}$.

The authors acknowledge support of the French Agence Nationale de la Recherche (ANR), through the programs "Investissements d'Avenir" LabEx EMC ${ }^{3}$ (ANR-10-LABX-09-01, MESOTHERM project) and IdEx Bordeaux - CPU (ANR-10-LABX-09-01). The laboratory LEGI is part of the LabEx Tec 21 (Investissements d'Avenir - grant agreement nANR-11-LABX-0030). This work was granted access to the HPC resources of IDRIS, CCRT and CRIHAN, under the allocations x20142b6186 and i20142a0611 (made by GENCI, Grand Equipement National de Calcul Intensif) and 2008013 (Centre de Ressources Informatiques de Haute-Normandie), respectively.

\section{REFERENCES}

1. Bose ST, Moin P, You D. Grid-independent large-eddy simulation using explicit filtering. Physics of Fluids 2010; 22(10):105-103.

2. Celik I, Klein M, Freitag M, Janicka J. Assessment measures for URANS/DES/LES: an overview with applications. Journal of Turbulence 2006; 7:48, doi:10.1080/14685240600794379.

3. Xing T. A general framework for verification and validation of large eddy simulations. Journal of Hydrodynamics 2015; 27.

4. Addad Y, Benhamadouche S, Laurence D. The negatively buoyant wall-jet: Les results. Int. J. Heat Fluid Flow $2004 ; 25$.

5. Gant SE. Reliability issues of les-related approaches in an industrial context. Flow Turbul. Combust. 2010; 84.

6. Klein M. An attempt to assess the quality of large eddy simulations in the context of implicit filtering. Flow Turbul. Combust. 2005; 75.

7. Freitag M, Klein M. An improved method to assess the quality of large eddy simulations in the context of implicit filtering. Journal of Turbulence 2006; 7. 
8. Celik IB, Cehreli ZN, Yavuz I. Index of Resolution Quality for Large Eddy Simulations. Journal of Fluids Engineering 2005; 127(5):949.

9. Celik I, Klein M, Janicka J. Assessment measures for engineering les applications. J. Fluids Eng. 2009; 131.

10. Geurts BJ, Frohlich J. A framework for predicting accuracy limitations in large-eddy simulation. Physics of Fluids 2002; 14(6):L41-L44, doi:http://dx.doi.org/10.1063/1.1480830.

11. Davidson L. How to estimate the resolution of an les of recirculating flow. Quality and Reliability of Large-Eddy Simulations II. Springer, 2011; 269-286.

12. Pope SB. Turbulent Flows. Cambridge Univ. Press, 2000.

13. Zhang B, Tong W, Gu CG, Dai ZY. An adaptive control strategy for proper mesh distribution in large eddy simulation. Journal of Hydrodynamics, Ser. B 2010; 22(6):865-870.

14. Löhner R, Baum JD. Adaptive h-refinement on 3d unstructured grids for transient problems. International Journal for Numerical Methods in Fluids 1992; 14(12):1407-1419.

15. Alauzet F, Frey P, George P, Mohammadi B. 3d transient fixed point mesh adaptation for time-dependent problems: Application to \{CFD\} simulations. Journal of Computational Physics 2007; 222(2):592 - 623, doi:http://dx. doi.org/10.1016/j.jcp.2006.08.012. URL http: / /www. sciencedirect.com/science/article/pii/ S002199910600369X.

16. Dompierre J, Vallet MG, Bourgault Y, Fortin M, Habashi WG. Anisotropic mesh adaptation: towards userindependent, mesh-independent and solver-independent CFD. Part III. Unstructured meshes. International Journal for Numerical Methods in Fluids 2002; 39(8):675-702.

17. Speares W, Berzins M. A 3d unstructured mesh adaptation algorithm for time-dependent shock-dominated problems. International Journal for Numerical Methods in Fluids 1997; 25(1):81-104.

18. Habashi WG, Dompierre J, Bourgault Y, Ait Ali Yahia D, Fortin M, Vallet MG. Anisotropic mesh adaptation: towards user-independent, mesh-independent and solver-independent CFD. Part I: general principles. International Journal for Numerical Methods in Fluids 2000; 32(6):725-744.

19. Ait Ali Yahia D, Baruzzi G, Habashi WG, Fortin M, Dompierre J, Vallet MG. Anisotropic mesh adaptation: towards user-independent, mesh-independent and solver-independent CFD. Part II. Structured grids. International Journal for Numerical Methods in Fluids 2002; 39(8):657-673.

20. Bedford KW, Yeo WK. Conjective filtering procedures in surface water flow and transport. Large Eddy Simulation of Complex Engineering and Geophysical Flows, edited by B. Galperin, and S. A. Orszag, Cambridge Univ. Press, New York 1993.

21. Naudin A. Simulation des grandes échelles de la combustion turbulente avec chimie détaillée tabulée. PhD Thesis 2008.

22. Deardorff J. Stratocumulus-capped mixed layers derived from a three-dimensional model. Bound.-Layer Meteor. 1980; 18:495-527.

23. Yoshizawa A, Horiuti K. A statistically-derived subgrid-scale kinetic energy model for the large-eddy simulation of turbulent flows. J. Phys. Soc. Jpn. 1985; 54. 
24. Lesieur M. Turbulence in fluids. Kluwer Academic Publisher, 1997.

25. Germano M, Piomelli U, Moin P, Cabot WH. A dynamic subgrid-scale eddy viscosity model. Phys. Fluids A 1991; 3:1760-1765.

26. Lilly D. A proposed modification of the Germano subgrid-scale closure method. Phys. Fluids A 1992; 4:633-635.

27. http://www.coria-cfd.fr.

28. Moureau V, Domingo P, Vervisch L. Design of a massively parrallel cfd code for complex geometries. $C$. $R$. Mecanique 339; (2/3):141-148.

29. Chorin AJ. Numerical solution of the navier-stokes equations. Mathematics of computation 1968; 22(104):745-762.

30. Pierce CD, Moin P. Progress-variable approach for large-eddy simulation of non-premixed turbulent combustion. Journal of Fluid Mechanics 2004; 504:73-97.

31. Malandain M, Maheu N, Moureau V. Optimization of the deflated conjugate gradient algorithm for the solving of elliptic equations on massively parallel machines. Journal of Computational Physics 2013; 238.

32. Moureau V, Domingo P, Vervisch L. From Large-Eddy Simulation to Direct Numerical Simulation of a lean premixed swirl flame: Filtered laminar flame-PDF modeling. Combustion and Flame Jul 2011; 158(7):1340-1357.

33. Guedot L, Lartigue G, Moureau V. Design of implicit high-order filters on unstructured grids for the identification of large scale features in les and application to a swirl burner. Physics of Fluids 2015; 27(045107).

34. da Silva $\mathrm{CB}$, Métais $\mathrm{O}$. On the influence of coherent structures upon interscale interactions in turbulent plane jets. J. Fluid Mech. 2002; 473:103-145.

35. Wu X, Moin P. A direct numerical simulation study on the mean velocity characteristics in turbulent pipe flow. $J$. Fluid Mech. 2008; 608.

36. Dobrzynski C, Frey P. Anisotropic delaunay mesh adaptation for unsteady simulations. Proc.of 17th Int. Meshing Roundtable, Pittsburgh, USA 2008.

37. Dapogny C, Dobrzynski C, Frey P. Three-dimensional adaptive domain remeshing, implicit domain meshing, and applications to free and moving boundary problems. Journal of Computational Physics 2014; 262(0):358-378, doi:http://dx.doi.org/10.1016/j.jcp.2014.01.005.

38. Ham F, Iaccarino G. Energy conservation in collocated discretization schemes on unstructured meshes. Annual Research Briefs 2004; 2004:3-14.

39. Karypis G, Kumar V. A parallel algorithm for multilevel graph partitioning and sparse matrix ordering. Journal of Parallel and Distributed Computing 1998; 48(1):71 - 95, doi:http://dx.doi.org/10.1006/jpdc.1997.1403.

40. Chevalier C, Pellegrini F. Pt-scotch : A tool for efficient parallel graph ordering. Parallel Computing 2008; 34(6):318-331.

41. Boudier G, Staffelbach G, Gicquel LM, Poinsot T. Mesh dependency of turbulent reacting large-eddy simulations of a gas turbine combustion chamber. Quality and Reliability of Large-Eddy Simulations, Ercoftac Series, vol. 12, Meyers J, Geurts B, Sagaut P (eds.). Springer Netherlands, 2008; 319-330.

42. Liu S, Renou B, Sjöstrand M, Yves D’Angelo FC. Experimental study of combustion and flow dynamics in a meso-scale whirl combustor. 15th Int Symp on Applications of Laser Techniques to Fluid Mechanics, 2010. 
43. Rivara MC. Mesh refinement processes based on the generalized bisection of simplices. SIAM Journal on Numerical Analysis 1984; 21(3):604-613.

44. Nicoud F, Ducros F. Subgrid-scale stress modelling based on the square of the velocity gradient tensor. Flow, Turbulence and Combustion 1999; 62(3):183-200.

45. Benard P, Moureau V, Lartigue G, D’Angelo Y. Large eddy simulation of a meso-scale combustion chamber. European Combustion Meeting, 2013.

46. Sjöstrand M. Simulations numériques directes d'une méso-chambre de combustion: Mise en oeuvre et analyses. PhD Thesis 2012.

47. Sjostrand M, D’Angelo Y. Dns analysis of a cubic meso-scale combustion chamber: I. cold flow topology \& dynamics. European Journal of Mechanics B/Fluids 2015; .

48. Hunt J, Wray A, Moin P. Eddies, stream, and convergence zones in turbulent flows. Center for turbulence research report CTR-S88 1988; :193-208. 